\title{
A precision analysis method for the kinematic assembly of complex products based on equivalence of deviation source
}

\author{
Dongping Zhao ${ }^{1,2}$, Gangfeng Wang ${ }^{2, *}$, Jizhuang Hui ${ }^{2}$, Wei Hou ${ }^{1}$, Richard Evans ${ }^{3}$
}

1 School of Aircraft Engineering, Xi' an Aeronautical University, Xi' an, China

2 Key Laboratory of Road Construction Technology and Equipment of MOE, School of Construction Machinery, Chang'an University, Xi'an, China

3 College of Engineering, Design and Physical Sciences, Brunel University London, London, United Kingdom

* Corresponding Author: Gangfeng Wang; E-mail: wanggf@chd.edu.cn

\begin{abstract}
:
Purpose - The assembly quality of complex products is pivotal to their lifecycle performance. Assembly Precision Analysis (APA) is an effective method used to check the feasibility and quality of assembly. However, there is still a need for a systematic approach to be developed for APA of kinematic mechanisms. In order to achieve more accurate analysis of kinematic assembly, a precision analysis method, based on equivalence of deviation source, is proposed in this paper. Design/methodology/approach - A unified deviation vector representation model is adopted by considering dimension deviation, geometric deviation, joint clearance, and assembly deformation. Then, vector loops and vector equations are constructed, according to joint type and deviation propagation path. A combined method, using deviation accumulation and sensitivity modeling, is applied to solve the kinematic APA of complex products.

Findings - When using the presented method, geometric form deviation, joint clearance, and assembly deformation, are considered selectively during tolerance modeling. In particular, the proposed virtual link model and its orientation angle are developed to determine joint deviation. Finally, vector loops and vector equations are modeled to express deviation accumulation.

Originality/value - The proposed method provides a new means for the APA of complex products, considering joint clearance and assembly deformation, while improving the accuracy of APA, as much as possible.
\end{abstract}

Keywords: Assembly precision analysis, Kinematic assembly, Deviation source, Equivalent analysis. 


\section{Introduction}

Assembly precision analysis is an important activity performed during complex product design, process planning and manufacture. Its aim is to evaluate the quality of assembly and the feasibility in design or process planning (Polini and Corrado 2015). As performance requirements for products increase, the required precision of assembly also amplifies. There is, therefore, a strong need for improved accuracy in APA to obtain high-precision assemblies at lower manufacturing costs (Geetha et al. 2015). With continuous improvement of assembly precision requirements, scholars have begun to explore the idea of precision control during the assembly design process. At present, methods of APA and simulation are used to predict and verify the accessibility of complex product assembly. However, existing methods typically deal with static assemblies or mechanisms that are treated as static assemblies, in which kinematic characteristics and assembly deformation are ignored. When these methods are applied to mechanisms or kinematic assembly, their performance may meet the design requirements, after completion of assembly, but the quality is often deemed poor.

It is commonly understood that many mechanisms exist in the assembly of a product, except for fixed or rigid parts. Numerous sources of deviation in complex product kinematic assembly exist, with assembly precision being affected by multiple deviation sources. In comparison to fixed or rigid assembly, the kinematic assembly of complex products has the following characteristics:

Assembly deformation and assembly joint clearance deviation cannot be ignored.

There are many connecting rods involved in kinematic assembly and deformation is obvious under gravity and assembly force. Assembly deformation has a significant influence on the precision of a final assembly. In addition, when kinematic assembly involves high precision and large sizes, such as in the case of an aircraft wing or landing gear, the requirement for assembly precision (especially motion precision) is high and the accumulation effect of the deviation on the transmission path cannot be ignored.

The amplification effect of deviation is obvious.

The influence of deviation sources on assembly precision is not only related to the deviation value, but also to their mechanism. There are many slender parts involved in the assembly of complex products; an obvious amplification effect of deviation exists, with a small deviation potentially having a severe impact on assembly precision.

The deviation direction is diverse.

During the kinematic assembly of complex products, the matching position of parts changes with the movement of the mechanism, which causes the deviation direction to change. In addition, the actual assembly position of the parts will deviate from the ideal position under the influence of a deviation source (such as assembly deformation, geometric deviation, etc.). This will lead to a change in deviation direction. Due to the diversity of deviation directions, the deviation transmission and accumulation for kinematic assembly has become more complicated. 
A large amount of kinematic assembly exists in complex products. This is not only required so that performance meets design requirements after assembly but, also so that the corresponding precision of motion is experienced during movement. High kinematic precision plays a significant role in the performance of the entire mechanism; APA is an effective method for ensuring its requirements (Sahani et al. 2017); hence, understanding how to develop a new method to analyze kinematic assembly precision, before manufacture, is seen as key to improving the quality of assembly and achieving higher assembly success rates for complex products.

The aim of this research paper is to propose a precision analysis method for kinematic assembly, based on equivalence of deviation source. Deviation modeling and kinematic APA have become accurate through equivalent of joint clearance, geometric form deviation and assembly deformation. The main outcomes of this paper include: (1) Geometric form deviation and assembly deformation are considered selectively in tolerance modeling; (2) Virtual link model and the orientation angle are developed to determine the joint angular deviation; and (3) Vector loops and equations are modeled to express deviation accumulation.

The remainder of this paper is organized as follows: in Section 2, related work about APA and deviation modeling methods is presented. In Section 3, we propose a precision analysis schema for kinematic assembly. Section 4 puts forth a unified vector representation and the corresponding equivalent method for multiple deviation sources. Section 5 presents the proposed approach of kinematic APA, based on equivalence of deviation source. Then, in Section 6, a case study is introduced to demonstrate the rationality of the proposed approach. Finally, conclusions and suggestions for future work are presented.

\section{Literature review}

With ever-increasing requirements in the assembly of complex products, APA methods have been developed from traditional one/two dimensional (1/2D) to three dimensional (3D) (Dantan and Qureshi 2009). 3D APA is an innovative method which represents deviation in the 3D space. The major advantage is that geometric deviation and dimensional deviation, as well as the interactions between them in the tolerance zone, can be considered. A substantial amount of research has been devoted to the development of 3D APA. Usually, APA includes two parts of assembly deviation representation and modeling: deviation propagation and accumulation calculation (Zhang et al. 2017; Cao et al. 2018). Representation or modeling methods for assembly deviation have been researched extensively, such as in the contexts of: Deviational geometry (Light and Gossard 1982), Virtual boundary (Srinivasan and Jayaraman 1989), Vectoral approach (Geis et al. 2015), Virtual joint modeling (Kramer 1993), Degree of Freedom (DOF) (Chase et al. 1995), Tolerance-Map (T-Map) (Mujezinovic et al. 2004; Davidson et al. 2012), Topologically and Technologically Related Surfaces (TTRS) (Desrochers and Clémentt 1994), Matrix (Whitney et al. 1994), The unified Jacobian-Torsor model (Corrado and Polini 2017), and Proportioned Assembly Clearance Volume (PACV) (Teissandier et al. 1999) etc. Deviation propagation and accumulation calculation describes the accumulation effect of deviation during assembly. It is determined using various 
methods, such as Direct Linearization Method (DLM) (Cao et al. 2018), Network of zones and datums (Peng and Wang 2017), Taguchi method (D'Errico and Zaino 1998), Monte Carlo simulation (Qureshi et al. 2012), Kinematic formulation (Rivest et al. 1994) and Jacobian matrix (Chen et al. 2015) etc. For statistical tolerance analysis, the input variables of deviation are continuous random variables. In general, they can be mutually dependent. A variety of methods and techniques, such as Root Sum of Squares, Extended Taylor series, Monte Carlo Simulation etc. are available to estimate the probability distribution of assembly precision control target.

Deviation sources of kinematic assembly include dimensional deviations, geometric form deviation, joint clearance and assembly deformation etc. Compared with general mechanical products, the difficulty resides in how to consider joint clearance and assembly deformation during kinematic APA. The related research is described against two aspects, as follows.

\subsection{APA methods considering joint clearance}

The above APA methods are applied to both static assemblies and kinematic assembly. However, these two bodies of literature remain somewhat separated, even though the analysis methods are quite similar (Chase and Parkinson 1991). In the context of APA of kinematic assembly, Howell, Larry and Jonathan (2004) used DLM for kinematic position error analysis. Sacks and Joskowicz (1998) introduced an algorithm for worst-case scenario for kinematic APA of general planar mechanical systems, which can be used to study kinematic deviation. Muvengei (2011) reviewed the evolution of APA, considering imperfect kinematic joints in the dynamic analysis of multi-body systems. However, these approaches do not require a determination of contact positions between parts, and a general representation of joints with clearance inside a precision analysis model is still not offered. Therefore, they cannot be applied simply to kinematic APA, which requires a necessary contact analysis (Bruyère et al. 2007). To overcome this challenge, researchers have devoted time to investigating the influence of joint clearance on kinematic assembly (Parenticastelli and Venanzi 2005). Recently, many methods have been studied for joint clearance modeling. Meijaard (2002) presented a continuous contact force model to deal with revolute joints; however, their model cannot be used for assembly-oriented precision analysis. There are two main areas of research on joint clearance modeling for assembly. Firstly, statistical method treats the joint clearance as a dimensional deviation (Beaucaire et al. 2013; Corrado et al. 2018; Tsai et al. 2008). However, the behavior of joint clearance in a kinematic assembly is different from the dimensional deviation. The second method treats the joint clearance by inserting a clearance vector or a small link into the functional relationship. Polini (2014) developed a method to model joint clearance by defining a possible movement between two components; however, the model was solely suited to rigid parts. Ting (2000) presented an approach to identify worst position and directional errors with joint clearance considered. Joint clearance was treated as a small link with the length equal to one half of the clearance. In this approach, a geometrical model was used to assess the output position or directional deviation to predict the limit of position uncertainty, to determine the maximum clearance and, ultimately, to assure assembly precision of a mechanism. 
This model can be used directly in any single closed-loop mechanism and can be extended to some multi-loop mechanisms. Tsai and Lai (2014) presented a method for error analysis of multi-loop mechanisms with joint clearance. Equivalent kinematical pairing was used to model the motion freedoms furnished by the joint clearances. Rhyu and Kwak (1998) formulated the problem of optimal design for mechanisms in the context of joint clearance, with an equivalent linkage model being presented. Erkaya (2009) and Rao (2004) used genetic algorithms and fuzzy theory to determine link parameters for minimizing the error between desired and actual paths due to clearance, but these cannot be used to estimate the deviations in the joint angle. Mallik et al. (1987) introduced a stochastic model of four-bar function generators. They considered deviations on link length and clearance in hinge joints as random variables and developed a Lagrange Multiplier approach to allocate tolerances and clearances. Lee and Gilmore (1991) introduced a model which considered the effect of link length deviation, radial clearance and uncertainty of pin location as a net effect on the link's effective length. However, their approach did not consider the deviation of joint angle in joint clearance modeling and this may lead to erroneous results. More recently, Zhao et al. (2018) presented an uncertainty analysis method of assembly error for planar single-loop mechanisms based on the rotatability laws of linkages. An uncertainty analysis model of assembly error of the planar single-loop mechanism was built with considered deviations of joint angle, and the maximum and minimum assembly error is given. However, this method studies the influence of joint clearance on assembly deviation and only considers the relative rotation of joint surfaces. In fact, due to joint clearance, the relative motion of joint surfaces is not only rotating, but also a coupled motion with rotating and translating occurs. Therefore, in equivalence of joint clearance, it is necessary to consider the influence of the coupling motion of the joint mating surface on the assembly position.

\subsection{APA methods considering assembly deformation}

Due to increasing quality requirements for complex products, there exists a growing interest in the consideration of geometric deviation and other deviations in kinematic APA. Walter et al. (2013) illustrated an approach for precision analysis with defined types of geometric deviations, as well as their interactions; the obtained results can be used for precision optimization (Lee and Tang 2000). Dantan et al. (2008) also used this approach for tolerance allocation. However, the approaches above do not provide adequate results when large deformation occurs. To overcome this problem, Finite-Element Analysis (FEA) is employed. Gerbino et al. (2008) proposed a linear methodology using Statistical Variation Analysis and Finite Element Analysis (SVA-FEA), in which a linear contact algorithm was implemented by using multipoint constraint elements of Nastran. Wang and Xie (2007) used a non-linear FEA approach to solve the deformation problem in APA; their method was more accurate than a linear one. In addition, Dupac and Beale (2010), Stuppy and Meerkamm (2009) also considered elastic deformation due to motion-induced forces. Imani (2009) used a DLM to analyze the kinematic precision of flexible mechanisms, but geometric deviation is not considered simultaneously in these two methods. A discrete geometry framework was proposed in 
some research (Wu et al. 2018; Schleich et al. 2014). In this framework, part representation with geometric deviation was depicted as Skin Model. Schleich (2014) also proposed an approach for mechanism precision analysis, based on discrete geometry representation of non-ideal parts. Franciosa et al. (2011) used a morphing mesh approach to analyze compliant assembly precision, considering shape errors. However, severe assumptions were made in these approaches that may not handle all types of 3D tolerance. Furthermore, they are very time consuming, especially if combined with Monte Carlo simulation.

From the review of previous work and the status of current technologies, it is observed that taking dimensional deviation, geometric deviation, assembly deformation and their interactions into account for kinematic APA will produce a more accurate result. Although some methods have been delved into and have their own advantages, joint angular deviation, geometric form deviation and assembly deformation have not been researched concretely in kinematic APA. Hence, we will focus on the equivalent of these deviations and how to represent them in tolerance modeling. Then, a vector loops model will be developed for kinematic APA.

\section{General outline of kinematic APA}

APA aims to evaluate the cumulative effect of deviations on the functional requirements. A functional requirement may be represented by an equation whose parameters are deviations. To establish equations of assembly, functional requirements and their parameters in kinematic assembly is difficult, because these parameters depend not only on the dimensional deviations, but also on geometric deviations, joint clearance, and assembly deformation etc. The proposed outline of precision analysis for kinematic assembly is shown in Figure 1; it includes several major phases of deviation source analysis, deviation representation and deviation accumulation calculation. 


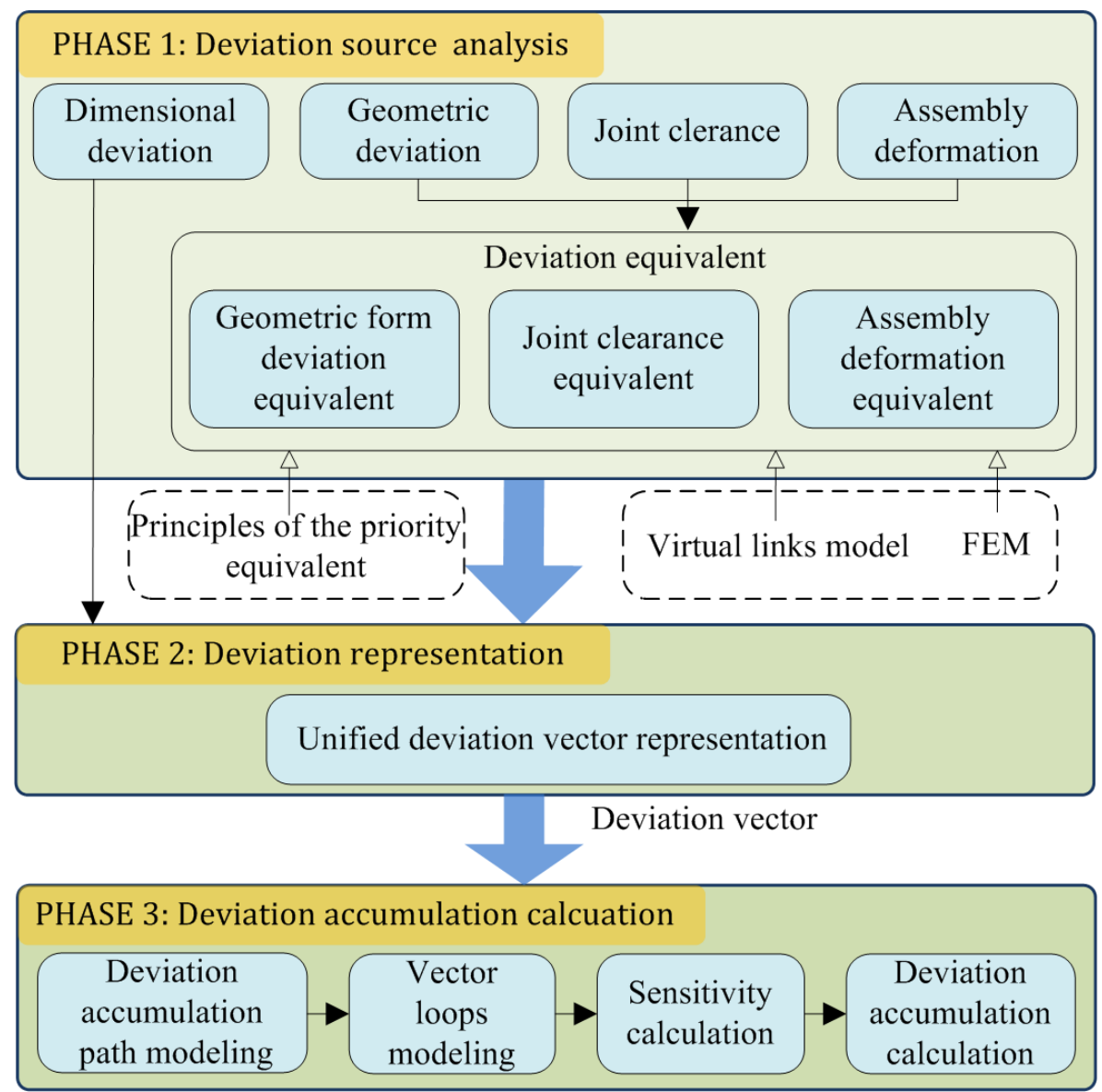

Figure 1 General outline of kinematic APA.

Phase 1. The virtual links model is established to describe the joint clearance of the kinematic assembly. Equivalent analysis methods of deviation source are proposed. Equivalent of geometric deviation, joint clearance, and assembly deformation are studied, based on priority equivalent principle, virtual links model and FEA.

Phase 2. In this phase, the deviation vector is introduced to represent each equivalent deviation, so that deviation of kinematic assembly can be expressed uniformly by deviation vector.

Phase 3. The deviation accumulation path is modeled, according to joint types of kinematic assembly. Then, vector loops and vector equations are established to describe the mechanism of deviation propagation and accumulation. Sensitivity of deviation source and deviation accumulation is also calculated.

\section{Equivalence of deviation source}

Joint clearance and hot force deformation etc. are also sources of deviation in kinematic assembly, besides those related to manufacturing deviations. Deviation source of kinematic assembly can be summarized as follow.

\section{Deviations in manufacturing}

These consist of dimensional and geometric deviations. Dimensional deviation includes linear, dimensional and angular deviations. Dimensional deviation $\delta R$ is shown in Figure $2(a)$. Geometric 
deviation includes geometric position deviation and geometric form deviation, such as flatness, cylindricity, straightness, profile of line and surface etc. For example, flatness deviation $\delta t$ is shown in Figure $2(a)$. Geometric deviation has a significant impact on APA since it is involved in deviation accumulation.

\section{Deviations in assembly}

These deviations are mainly introduced by joint clearances and assembly deformation. Joint clearance contains constant and random deviations. The former is given by the designer to meet the performance requirements of the kinematic property. The latter is introduced by joint clearance. Examples of $\delta u$ and $\delta r$ are shown in Figure $2(a)$ and $(b)$. Deformations in assembly include force deformation, locating and clamping deformation, etc. For example, clamping deformation $\delta v$ is shown in Figure $2(c)$.

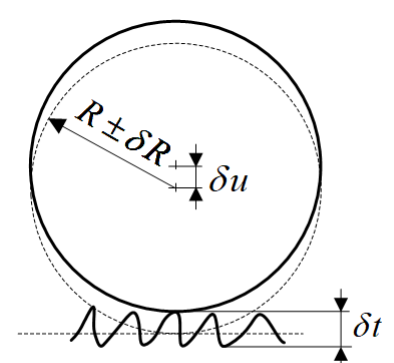

(a)

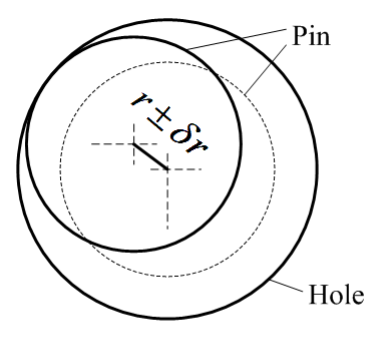

$(b)$

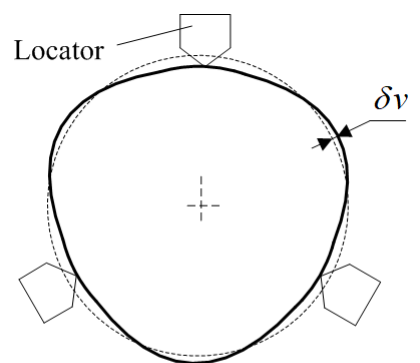

(c)

Theoretical position

Figure 2 Deviation examples of kinematic assembly.

As deviations of geometric form, joint clearance and assembly deformation cannot be expressed directly and accurately in an APA model, the equivalents of these deviations are needed. Therefore, this section is focused on the equivalent of these three types of deviation.

\subsection{Equivalence of geometric form deviation}

Geometric form deviation restricts the range of geometric features and is described by tolerance zone. Due to the actual shape of the mating surface being waveform, as shown in Figure 2 (a), existing precision analysis methods rarely consider geometric form deviation. Therefore, there is a need to handle the mating surface by considering geometric form deviation. Compared to other sources of deviation, geometric form deviation is smaller and has little effect on deviation accumulation. However, there is great influence when in a specific direction. Therefore, it is necessary to make a priority before equivalent of geometric form deviation. The principles of the priority are described as follows.

(1) Deviations are not considered in deviation accumulation, when their directions are perpendicular to the deviation accumulation direction. For example, the circularity of part 2 in Figure 3 has no influence on deviation accumulation and it need not be considered.

(2) Only one deviation is considered in deviation accumulation when several deviations are defined on the same feature, such as location, orientation, and shape deviation. In particular, positional deviation has the highest priority, orientation deviation is second, and form 
deviation is last. In Figure 3, only parallelism of the up surface on part 1 is considered in deviation accumulation.
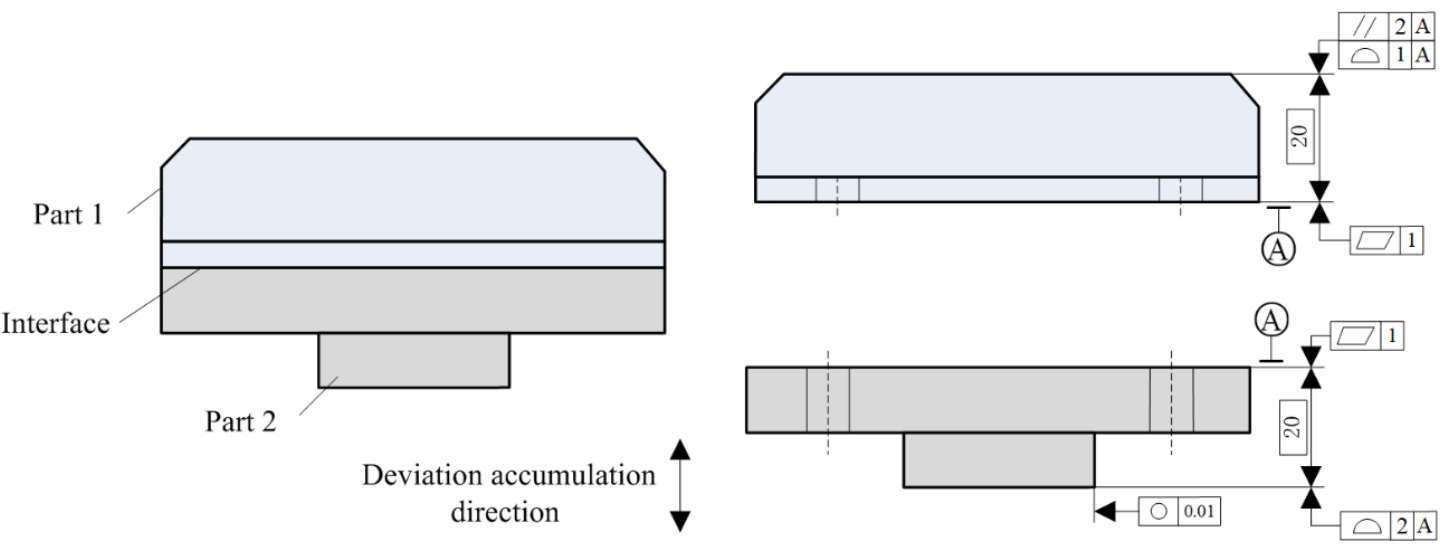

Figure 3 An example of geometric form deviations.

Geometric form deviation can cause the mating position to change and introduce mating positional deviation to the assembly system. Influences of flatness deviation on the mating position are shown in Figure 4.

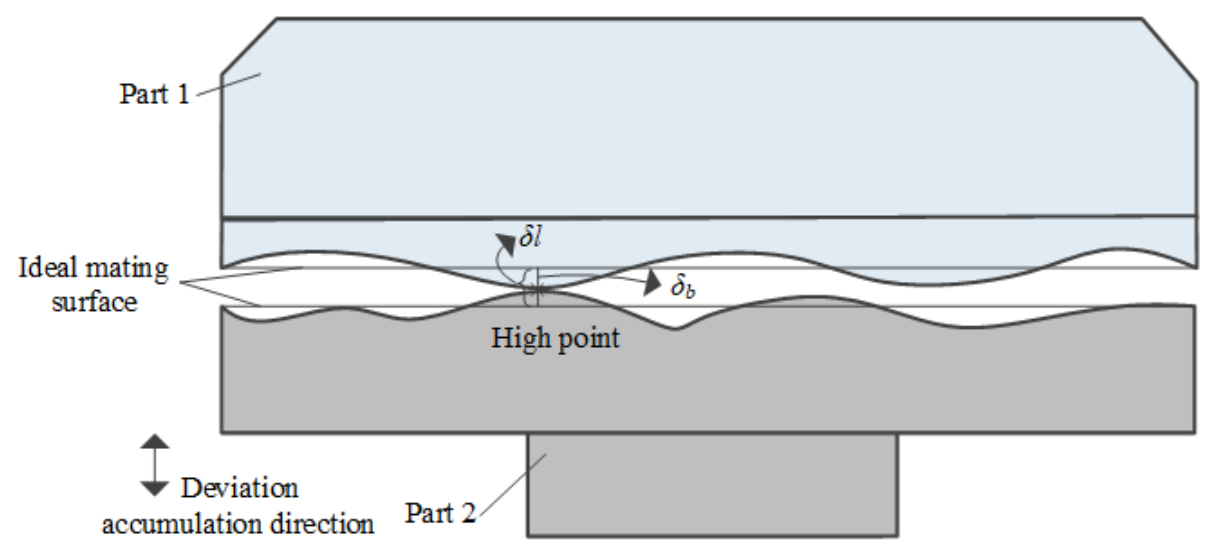

Figure 4 The influences of flatness deviation on the mating position.

Due to the variations and irregularities in manufacturing process, peaks or high points stand out from the surrounding part surface. These high points adequately represent a part feature's mutability. In the practical mating of two planes, parts contact each other only at a few microscopic points (high points) (Goka et al. 2019). Thus, the assembly deviation $\delta l$ is introduced, and it can be expressed by offset of the high point. The offset of the high point is marked as $\delta b$ in Figure 4.

Furtherly, the offset of high point can be equivalent to a dimensional deviation. In this way, flatness deviation can be expressed in the deviation propagation model and considered in deviation accumulation. Assumed that one of the mating surface's tolerance zone is $t$. Then, the offset of the high point is $t / 2$ approximately. In this situation, a geometry form deviation can be equivalent to a dimensional deviation where the nominal value is zero and the deviation is half of 
the tolerance zone. For example, In Figure 3, one of a flatness deviation is equivalent to a dimensional deviation $0 \pm t / 2$ ( $t$ is tolerance zone), that is $0 \pm 0.5$, and the direction is along with its tolerance zone.

\subsection{Equivalence of joint clearance}

Joint clearance has an impact not only on dimensional deviation, but also on the positional deviation of joints. Therefore, it can be equivalent to a dimensional deviation where direction is changed with the mating position.

Links $i$ and $j$ are mated by a revolute pair with joint clearance in Figure 5 (a). The rotation centers of the two links are in the same point when there is no clearance in the joint. However, because of joint clearance, there is a gap $\boldsymbol{r}_{i j}$ between the two rotation centers in the actual assembly. $\boldsymbol{r}_{i j}$ is defined as clearance ring radius. A virtual link which has no mass is used to replace the clearance ring radius. Then, the virtual link model of joint clearance for kinematic assembly is constructed in Figure $5(b)$. The dimension and deviation of virtual link can be solved by:

$$
r_{i j}+\delta r_{i j}=\left(r_{i}+\delta r_{i}\right)-\left(r_{j}+\delta r_{j}\right)
$$

Where $\boldsymbol{r}_{\boldsymbol{i} j}$ is the dimension of the virtual link, and $\boldsymbol{\delta} \boldsymbol{r}_{i j}$ is its deviation. $\boldsymbol{r}_{\boldsymbol{i}}$ is the mating radius of link $i$, and $\boldsymbol{\delta} \boldsymbol{r}_{i}$ is its deviation. $\boldsymbol{r}_{j}$ is the mating radius of link $j$, and $\boldsymbol{\delta} \boldsymbol{r}_{j}$ is its deviation.

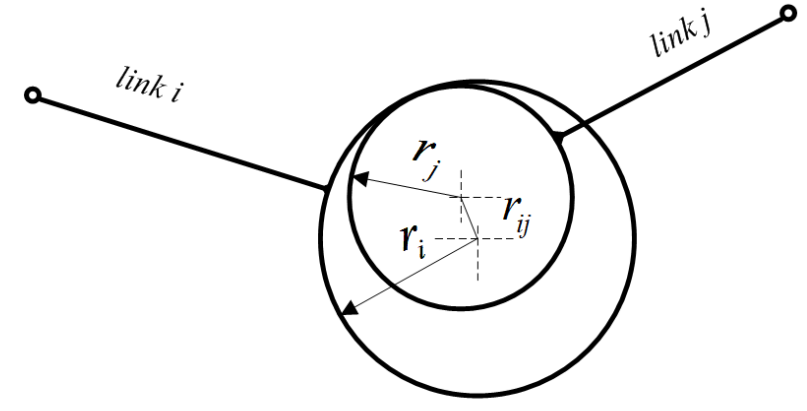

(a)

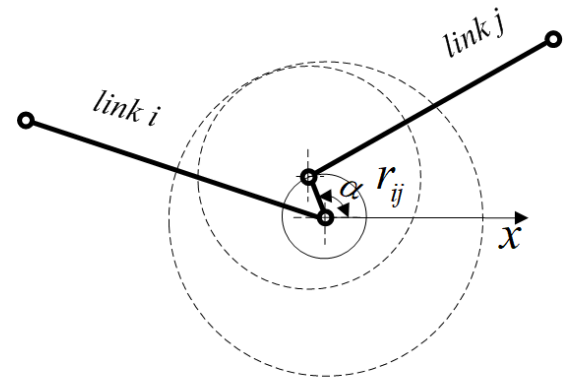

(b)

Figure 5 Virtual link model of joint clearance.

The angle $\alpha$ between $\boldsymbol{r}_{i j}$ and $\mathrm{x}$-axis is defined as orientation angle of the virtual link. $\alpha$ is used to describe the position of the virtual link. Therefore, the mating position of the parts in a joint can be determined and expressed by $\alpha$. There are two situations to obtain the orientation angle $\alpha$ of the virtual link.

\section{Rotation}

The position of link $i$ is represented by the dotted line in Figure $6(a)$ when link $i$ is turned with $\theta$ and joint clearance is not considered. In this situation, the rotation center of link $i$ is located at point $O$. The rotational speed $v_{1}$ and theoretical position of link $i$ are mutually orthogonal, according to the property of kinematics. If joint clearance is considered, link $i$ translates into a distance that is equivalent to joint clearance along $\boldsymbol{v}_{\mathbf{1}}$, and then starts to rotate around the fixed axis. In this situation, the position of link $i$ is represented by a solid line and the rotation center is located at point $A$. The position vector $\boldsymbol{r}_{i j}$ of the virtual link and theoretical position of link $i$ are mutually orthogonal. 
Therefore, the orientation angle $\alpha$ of the virtual link can be described as follows:

$$
\alpha=\theta \pm 90^{\circ}
$$

Where a positive sign is taken when link $i$ is turned counterclockwise with $\theta$, and a negative sign is taken when reversed.

\section{Motion with rotation and translation}

In Figure $6(b)$, a compound motion of link $i$ can be divided into fixed-axis rotation around $O$ and translation along with its axis. Due to the joint clearance, the rotation center of link $i$ is located at point $B$ when it is only present as a fixed-axis rotation. In this situation, the position vector $\boldsymbol{r}_{\mathbf{1}}$ of the virtual link and theoretical position of link $i$ are mutually orthogonal. In another respect, the rotation center is located at point $C$ when it only presents a translation. In this situation, the position vector $\boldsymbol{r}_{2}$ of the virtual link is shown along with translational speed $\boldsymbol{v}_{\mathbf{2}}$. Therefore, the position vector $\boldsymbol{r}_{\boldsymbol{i j}}$ of the virtual link is synthesized by $\boldsymbol{r}_{1}$ and $\boldsymbol{r}_{2}$ when it presents a compound motion. The orientation angle $\alpha$ of the virtual link can be described as follows:

$$
\alpha=\theta \pm 45^{\circ}
$$

Where a positive sign is taken when link $i$ is rotated counterclockwise with $\theta$, and a negative sign is taken when reversed.

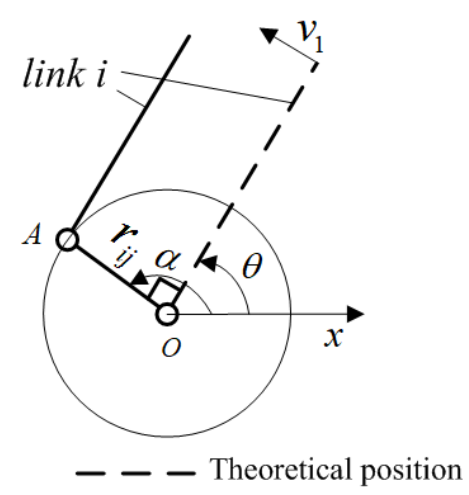

(a)

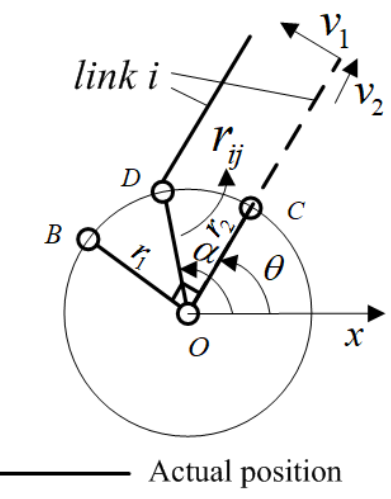

(b)

Figure 6 Orientation angles of the virtual link.

So far, joint clearance is equivalent to dimensional and angular deviations of the virtual link, based on the virtual link model.

\subsection{Equivalence of assembly deformation}

Assembly deformation is introduced by assembly force, gravity and other factors present in the assembly system. This has a major impact on kinematic assembly precision. Part dimension and assembly position are impacted by assembly deformation.

\section{Influences of assembly deformation on dimensions}

The main parts are links in the kinematic assembly. Assembly deviation is propagated and accumulated along the length direction of a link, which affects the assembly precision of the final assembly. Therefore, the axial deformation of a link will introduce dimension deviation. The direction of the deviation is along the length of the link. Design dimension is expressed with nominal value $L$ and deviation $T$ as shown in Figure $7(a)$. The allowable values of dimension in 
maximum and minimum are $L+T$ and $L-T$. An axial deformation is generated by a load $F$ as shown in Figure $7(b)$. Assuming the amount of deformation is $\delta$ on axis (tension is positive and pressure is negative), the maximum and minimum dimensions along with axis are $L+T+\delta$ and $L-T+\delta$.

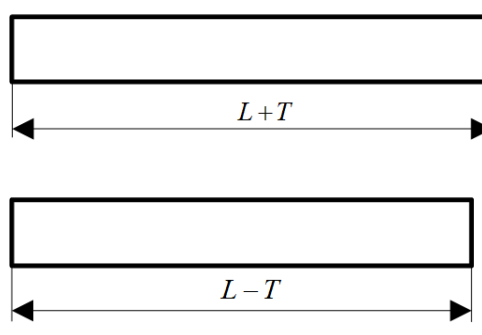

(a)

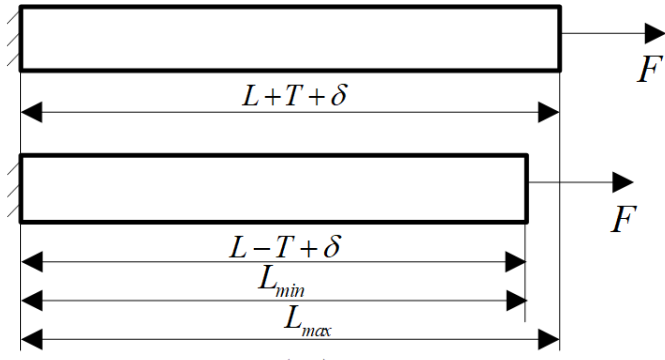

(b)

Figure 7 The influences of assembly deformation on the part's axial dimension.

Dimension and deviation in the direction of deformation are replaced by:

$$
\begin{aligned}
& L^{\prime}=\frac{L_{\max }+L_{\min }}{2} \\
& T^{\prime}=\frac{L_{\max }-L_{\min }}{2}
\end{aligned}
$$

where $L_{\max }$ is the maximum dimension in the direction of deformation. $L_{\min }$ is the minimum dimension in the direction of deformation.

\section{Influences of assembly deformation on assembly position}

Assembly deformation has an impact on the mating position of parts. The deformation under non-axial force will affect the position and/or dimension of the mating part. This influence on position and dimension can be equivalent to an angle deviation and/or a dimension deviation.

There is a hinge motion mechanism presented in Figure 8. The link with nominal dimension $L$ is turned with $\theta$ around the hinge. The assembly position is represented by a solid line if assembly deformation is not considered, and the actual assembly position is represented by the dotted line when gravity is considered. The amount of deformation $\delta u$ on axial and $\delta v$ on radial direction are solved by Finite Element Method (FEM). Where, $\delta u$ effects dimension $L$ it can be determined by equations (4) or (5). $\delta v$ effects assembly position. It is determined by $\Delta \theta$ as follow:

$$
\Delta \theta=\arctan \frac{\delta v}{L}
$$

where $\theta$ is a theoretical assembly position, in which assembly deformation is not considered. $\Delta \theta$ is an angular deviation that is introduced by assembly deformation. 


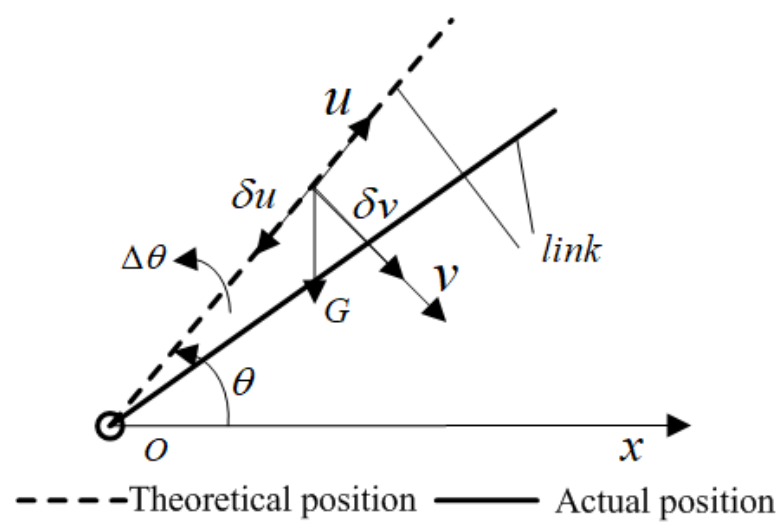

Figure 8 Assembly positions of hinge motion mechanism.

Therefore, deviations that are caused by assembly deformation on axis are calculated by equations (4) and (5) and on non-axis direction are calculated by equation (6).

\subsection{Unified deviation vector representation}

Deviations of geometric form deviation, joint clearance and assembly deformation are equivalent to parts' dimensional and angular deviations through the above analysis. Then, deviation vector is introduced to represent deviations in tolerance modeling. And a unified deviation vector representation is formed. For example, deviation $\boldsymbol{E}$ can be represented by vector, as follows:

$$
\boldsymbol{E}=(x, y, z, \alpha, \beta, \gamma)
$$

where $\boldsymbol{E}$ is a deviation vector. $x, y, z$ are values of the deviation vector. $\alpha, \beta, \gamma$ are directions of the deviation vector.

\section{Kinematic APA based on vector loops model}

As shown in Figure 1, APA includes several steps of deviation accumulation path modeling, vector loop modeling, sensitivity calculation and Monte Carlo simulation. The prior three steps are challenging for APA. The following sections focus on these three steps.

\subsection{Deviation accumulation path modeling}

Parts are identified by locating datum during the process of kinematic assembly. The locating datum is called Datum Reference Frame (DRF). The path from joint to the DRF is called Datum Path (DP). DP is represented by dimensional vector. A deviation accumulation path of two constrained parts is along a DP and through a joint. It needs to meet the following conditions: (a) enter a part through a joint; (b) follow the DP to the DRF in the part; (c) follow a second DP to another joint; and (d) exit the next part from the joint.

A deviation accumulation path is illustrated in Figure 9. J (Part1, Part2) is the joint of Part 1 and Part 2. There are four DPs in Part 1 and Part $2\left(\mathrm{DP}_{11}, \mathrm{DP}_{12}, \mathrm{DP}_{21}\right.$, and $\left.\mathrm{DP}_{22}\right)$. They are created by dimensional vectors $(a, b, c, d, e, f, g$, and $h)$. Dimension vector is the vector sum by nominal dimensional vector plus deviation vector. 


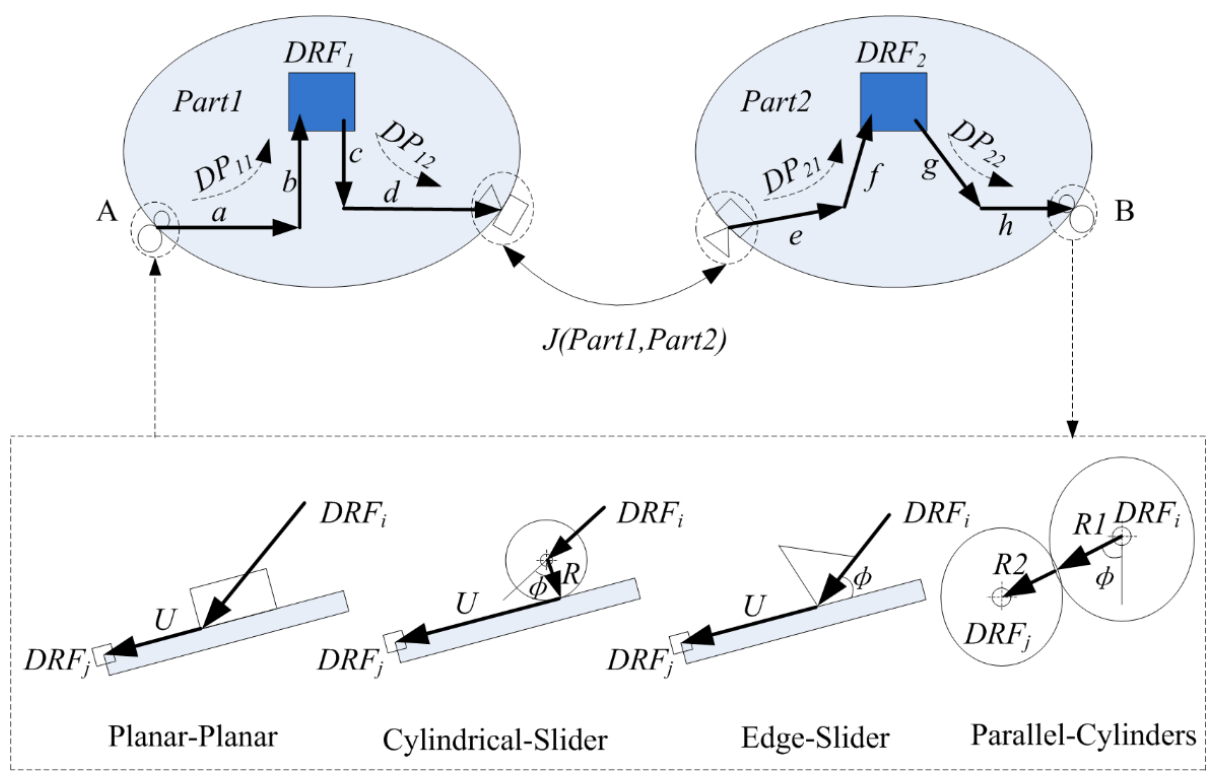

Figure 9 Deviation accumulation paths based on four kinds of joints.

\subsection{Vector loops and vector equation modeling}

The propagation path model of assembly deviation is constructed in Section 5.1, while the deviations in kinematic assembly are propagated and accumulated along with the propagation path. By combining the deviation vector expression in Section 4, the vector loop and vector equation of deviation propagation and accumulation are constructed.

Vector loops are expressed by dimension vector together with their accumulation paths, according to assembly constraints. They contain closed vector loops and opened vector loops. Closed vector loops describe relations between each deviation source. Opened vector loops describe relations between deviation source and functional requirement in the assembly. Rules for vector loop modeling are listed as follow:

(1) Vector loops must pass through every part and every joint in the assembly;

(2) A vector loop should pass through the same joint no more than once. However, it may start and end in the same part; and

(3) The dimensional vector must be omitted if a vector loop includes the same dimension vector twice, and their directions are opposite.

The number of closed vector loops can be expressed as:

$$
K=J-I+1
$$

where $K$ is the number of closed vector loops. $J$ is the number of joints. $I$ is the number of parts.

Based on the above principles, the multi-dimensional vector loop of deviation accumulation is established. In order to realize the mathematical expression of deviation accumulation, it is necessary to establish the deviation accumulation vector equation.

Assuming that $\left\{\boldsymbol{a}_{1}, \boldsymbol{a}_{2}, \cdots, \boldsymbol{a}_{n}\right\},\left\{\boldsymbol{b}_{1}, \boldsymbol{b}_{2}, \cdots, \boldsymbol{b}_{\boldsymbol{k}}\right\},\left\{\boldsymbol{r}_{1}, \boldsymbol{r}_{2}, \cdots, \boldsymbol{r}_{l}\right\}$ and $\left\{\boldsymbol{u}_{1}, \boldsymbol{u}_{2}, \cdots, \boldsymbol{u}_{\boldsymbol{m}}\right\}$ are vectors of the parts' dimension, virtual link dimension, geometric form deviation and subassembly 
dimension in vector loop. Then, vector equation can be expressed with a concatenation of homogeneous transformation matrix, as follows.

$$
[H]=\left[\begin{array}{llll}
\boldsymbol{a}_{1} & \boldsymbol{a}_{2} & \cdots & \boldsymbol{a}_{n}
\end{array}\right]^{T} \cdot\left[\begin{array}{lllll}
\boldsymbol{b}_{1} & \boldsymbol{b}_{2} & \cdots & \boldsymbol{b}_{k}
\end{array}\right]^{T} \cdot\left[\begin{array}{llll}
\boldsymbol{r}_{1} & \boldsymbol{r}_{2} & \cdots & \boldsymbol{r}_{l}
\end{array}\right]^{T} \cdot\left[\begin{array}{llll}
\boldsymbol{u}_{1} & \boldsymbol{u}_{2} & \cdots & \boldsymbol{u}_{m}
\end{array}\right]^{T}
$$

$\boldsymbol{H}$ is the vector matrix of functional requirements.

Equation (9) is decomposed in the coordinate direction and then,

$$
\boldsymbol{h}=S\left(\boldsymbol{a}_{1}, \boldsymbol{a}_{2}, \cdots, \boldsymbol{a}_{n}, \boldsymbol{b}_{1}, \boldsymbol{b}_{2}, \cdots, \boldsymbol{b}_{\boldsymbol{k}}, \boldsymbol{r}_{1}, \boldsymbol{r}_{2}, \cdots, \boldsymbol{r}_{l}, \boldsymbol{u}_{1}, \boldsymbol{u}_{2}, \cdots, \boldsymbol{u}_{m}\right)
$$

where, $\boldsymbol{h}$ is the assembly functional requirements vector in vector loop. $\boldsymbol{S}$ represents direction vector. $\boldsymbol{S}=(u, v, w, \alpha, \beta, \gamma)$ is in space and $\boldsymbol{S}=(x, y, \theta)$ is in plane. $u, v, w, x, y$ are positional parameters and $\alpha, \beta, \gamma, \theta$ are direction parameters of the vector.

In order to obtain the closed-loop and open-loop vector equation of deviation accumulation, the vector-loop equation (10) is decomposed on the closed-loop vector direction $\Lambda$ and the open-loop vector direction $\Gamma$, shown as follows.

$$
\left\{\begin{array}{l}
\boldsymbol{h}_{c}=\Lambda\left(\boldsymbol{a}_{1}, \boldsymbol{a}_{2}, \cdots, \boldsymbol{a}_{n}, \boldsymbol{b}_{1}, \boldsymbol{b}_{2}, \cdots, \boldsymbol{b}_{k}, \boldsymbol{r}_{1}, \boldsymbol{r}_{2}, \cdots, \boldsymbol{r}_{l}, \boldsymbol{u}_{1}, \boldsymbol{u}_{2}, \cdots, \boldsymbol{u}_{m}\right)=\mathbf{0} \\
\boldsymbol{h}_{\boldsymbol{o}}=\Gamma\left(\boldsymbol{a}_{1}, \boldsymbol{a}_{2}, \cdots, \boldsymbol{a}_{n}, \boldsymbol{b}_{1}, \boldsymbol{b}_{2}, \cdots, \boldsymbol{b}_{\boldsymbol{k}}, \boldsymbol{r}_{1}, \boldsymbol{r}_{2}, \cdots, \boldsymbol{r}_{l}, \boldsymbol{u}_{1}, \boldsymbol{u}_{2}, \cdots, \boldsymbol{u}_{m}\right)
\end{array}\right.
$$

where $\boldsymbol{\Lambda}$ represents closed-loop vector direction in accumulation. $\boldsymbol{\Gamma}$ represents an opened loop vector direction in accumulation. $\boldsymbol{h}_{\boldsymbol{C}}$ and $\boldsymbol{h}_{\boldsymbol{O}}$ are dimension vectors of assembly functional requirements in $\boldsymbol{S}$ and $\boldsymbol{\Gamma}$ direction.

\subsection{Calculation of sensitivity and assembly precision}

Vector equations can be linearized using Taylor's series expansion. The first-order Taylor's Series of closed and opened vector equations are expressed as follows:

$$
\left\{\begin{array}{l}
\delta h_{C}=\frac{\partial h_{C}}{\partial a_{1}} \delta a_{1}+\cdots+\frac{\partial h_{C}}{\partial a_{n}} \delta a_{n}+\frac{\partial h_{C}}{\partial b_{1}} \delta b_{1}+\cdots+\frac{\partial h_{C}}{\partial b_{k}} \delta b_{k}+\frac{\partial h_{C}}{\partial r_{1}} \delta r_{1}+\cdots+\frac{\partial h_{C}}{\partial r_{l}} \delta r_{l}+\frac{\partial h_{C}}{\partial u_{1}} \delta u_{1}+\cdots+\frac{\partial h_{C}}{\partial u_{m}} \delta u_{m}=0 \\
\delta h_{O}=\frac{\partial h_{O}}{\partial a_{1}} \delta a_{1}+\cdots+\frac{\partial h_{O}}{\partial a_{n}} \delta a_{n}+\frac{\partial h_{O}}{\partial b_{1}} \delta b_{1}+\cdots+\frac{\partial h_{O}}{\partial b_{l}} \delta b_{k}+\frac{\partial h_{o}}{\partial r_{1}} \delta r_{1}+\cdots+\frac{\partial h_{O}}{\partial r_{l}} \delta r_{l}+\frac{\partial h_{O}}{\partial u_{1}} \delta u_{1}+\cdots+\frac{\partial h_{O}}{\partial u_{m}} \delta u_{m}
\end{array}\right.
$$

where $\delta \boldsymbol{h}_{\boldsymbol{C}}$ is dimension deviation of closed-loop. $\delta \boldsymbol{h}_{\boldsymbol{O}}$ is dimension deviation of opened loop. $\delta \boldsymbol{a}_{\boldsymbol{n}}$ is deviation of dimension $\boldsymbol{a}_{\boldsymbol{n}} . \delta \boldsymbol{b}_{\boldsymbol{k}}$ is geometric form deviation. $\delta \boldsymbol{r}_{l}$ is deviation of virtual link dimension $\boldsymbol{r}_{\boldsymbol{l}} . \delta \boldsymbol{u}_{\boldsymbol{m}}$ is deviation of dimension $\boldsymbol{u}_{\boldsymbol{m}}$.

Closed-loop scalar equation of kinematic assembly is described as follows:

$$
\left[A_{1}\right][\delta a]+\left[B_{1}\right][\delta b]+\left[R_{1}\right][\delta r]+\left[U_{1}\right][\delta u]=0
$$

Opened loop scalar equation of kinematic assembly is described as follows:

$$
\left[\delta H_{O}\right]=\left[A_{2}\right][\delta a]+\left[B_{2}\right][\delta b]+\left[R_{2}\right][\delta r]+\left[U_{2}\right][\delta u]
$$

$\left[\boldsymbol{A}_{1}\right],\left[\boldsymbol{B}_{1}\right],\left[\boldsymbol{R}_{1}\right]$ and $\left[\boldsymbol{U}_{1}\right]$ are partial derivative matrixes of closed loop scalar function to part' 'dimensions, geometric form deviation, virtual link dimensions and subassembly dimensions. $\left[\boldsymbol{A}_{2}\right]$, 
$\left[\boldsymbol{B}_{2}\right],\left[\boldsymbol{R}_{2}\right]$ and $\left[\boldsymbol{U}_{2}\right]$ are partial derivative matrixes of opened loop scalar function to part' dimensions, geometric form deviation, virtual link dimensions and subassembly dimensions. Where,

$$
\begin{gathered}
{\left[A_{1}\right]=\left[\frac{\partial h_{C}}{\partial a_{i}}\right], \quad\left[B_{1}\right]=\left[\frac{\partial h_{C}}{\partial b_{i}}\right], \quad\left[R_{1}\right]=\left[\frac{\partial h_{C}}{\partial r_{i}}\right], \quad\left[U_{1}\right]=\left[\frac{\partial h_{C}}{\partial u_{i}}\right]} \\
{\left[A_{2}\right]=\left[\frac{\partial h_{O}}{\partial a_{i}}\right], \quad\left[B_{2}\right]=\left[\frac{\partial h_{O}}{\partial b_{i}}\right], \quad\left[R_{2}\right]=\left[\frac{\partial h_{O}}{\partial r_{i}}\right], \quad\left[U_{2}\right]=\left[\frac{\partial h_{O}}{\partial u_{i}}\right]}
\end{gathered}
$$

$\left[\delta \boldsymbol{H}_{o}\right]$ is dimension deviation matrix of opened loop. $[\delta \boldsymbol{a}],[\delta \boldsymbol{b}]$ are dimension and geometric form deviation matrixes of parts. $[\delta \boldsymbol{r}]$ is dimension deviation matrix of virtual link. $[\delta \boldsymbol{u}]$ is dimension deviation of subassembly.

$[\delta \boldsymbol{u}]$ can be solved from equation (13),

$$
[\delta u]=-\left[U_{1}^{-1}\right]\left[A_{1}\right][\delta a]-\left[U_{1}^{-1}\right]\left[B_{1}\right][\delta b]-\left[U_{1}^{-1}\right]\left[R_{1}\right][\delta r]
$$

Take the above formula into equation (14), and the functional requirement can be solved as follows.

$$
\begin{aligned}
{\left[\delta h_{o}\right] } & =\left[A_{2}\right][\delta a]+\left[B_{2}\right][\delta b]+\left[R_{2}\right][\delta r]+\left[U_{2}\right]\left(-\left[U_{1}^{-1}\right]\left[A_{1}\right][\delta a]-\left[U_{1}^{-1}\right]\left[B_{1}\right][\delta b]-\left[U_{1}^{-1}\right]\left[R_{1}\right][\delta r]\right) \\
& =\left(\left[A_{2}\right]-\left[U_{2}\right]\left[U_{1}^{-1}\right]\left[A_{1}\right]\right)[\delta a]+\left(\left[B_{2}\right]-\left[U_{2}\right]\left[U_{1}^{-1}\right]\left[B_{1}\right]\right)[\delta b]+\left(\left[R_{2}\right]-\left[U_{2}\right]\left[U_{1}^{-1}\right]\left[R_{1}\right]\right)[\delta r]
\end{aligned}
$$

where $\left[A_{2}\right]-\left[U_{2}\right]\left[U_{1}^{-1}\right]\left[A_{1}\right],\left[B_{2}\right]-\left[U_{2}\right]\left[U_{1}^{-1}\right]\left[B_{1}\right]$ and $\left[R_{2}\right]-\left[U_{2}\right]\left[U_{1}^{-1}\right]\left[R_{1}\right]$ are sensitivity matrices of parts' dimensions, geometric form deviation and virtual link dimensions.

Thus, the vector equation of assembly deviation accumulation calculation can be constructed, and the precision prediction of assembly function requirements can be obtained using the above equations.

\section{Case study and discussion}

The kinematic assembly of an aircraft wing flap was taken as an example to demonstrate APA, based on equivalence of deviation source. The process of demonstration includes several steps in presenting equivalent deviation source, unified deviation vector representation, vector loop modeling and deviation accumulation calculation.

The wing flap is an important component of an aircraft. The precision of its deflection has great influence on an aircraft's lift performance and kinematic APA is an effective means to deal with this problem. The assembly model of a wing flap is shown in Figure 10 (a). It includes parts of $\mathrm{P}_{\text {wing flap }}$, $\mathrm{P}_{\text {rocker }}, \mathrm{P}_{\text {link }}, \mathrm{P}_{\text {font connector }}, \mathrm{P}_{\text {middle connector }}$ and $\mathrm{P}_{\text {back connector. }} \mathrm{P}_{\text {link }}$ provides power to drive $\mathrm{P}_{\text {rocker }}$, and then

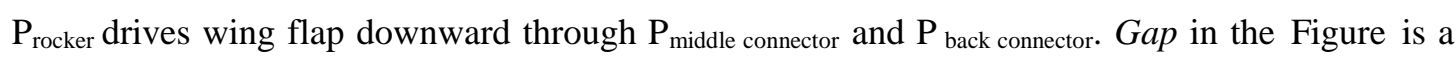
functional requirement for the assembly. It is a vertical distance deviation between $\mathrm{P}_{\text {wing body }}$ and the front of $\mathrm{P}_{\text {wing flap }}$ when wing flap is folded (the angle is $60^{\circ}$ between $\mathrm{P}_{\text {link }}$ and the negative direction of 
$x$ axis at the moment). The design dimensions and tolerances of the wing flap are shown in Figure 10 (b) and Table 1.

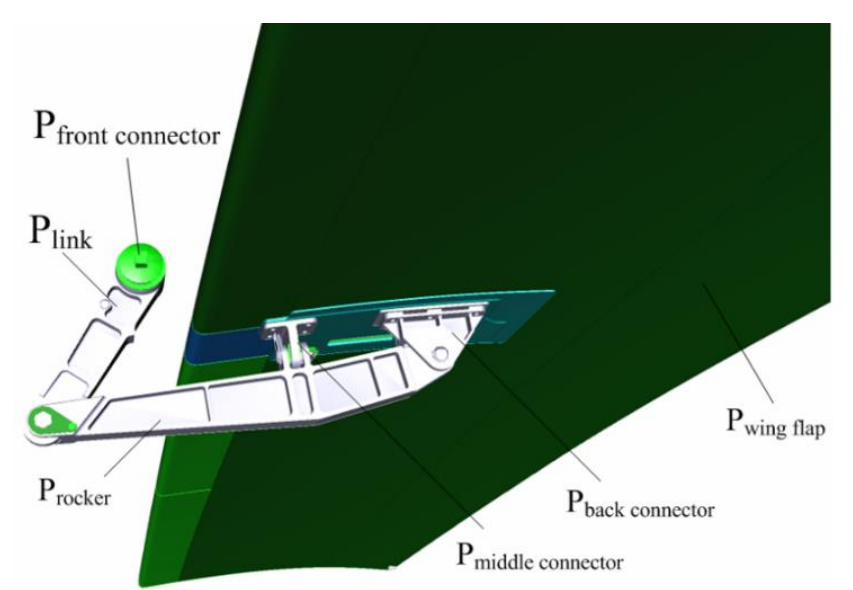

(a) 3D kinematic model of wing flap

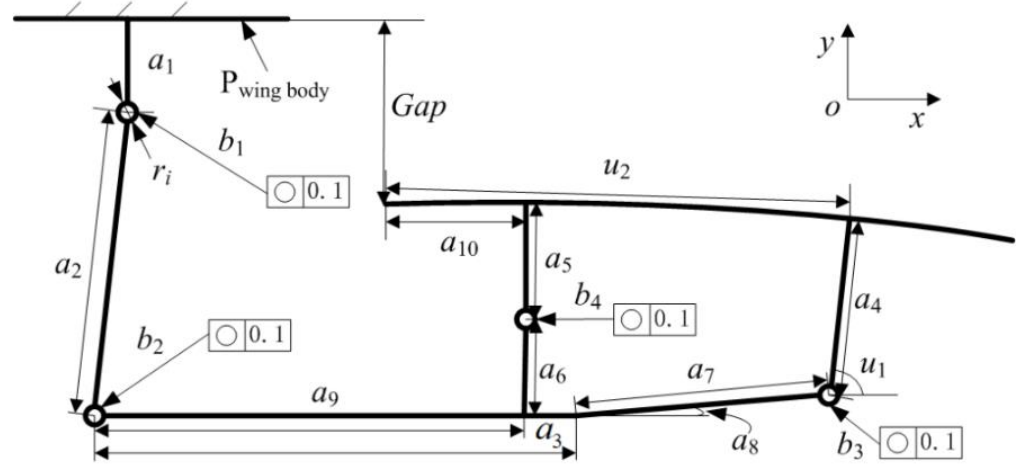

(b) Profile of wing flap kinematic assembly

Figure 10 The assembly model of wing flap.

Table 1 Nominal values and tolerances of design (Unit: $\mathrm{mm}$ ).

\begin{tabular}{cccccccccccccc}
\hline Parameters & $a_{1}$ & $a_{2}$ & $a_{4}$ & $a_{5}$ & $a_{6}$ & $a_{3}$ & $a_{7}$ & $a_{8}$ & $a_{9}$ & $a_{10}$ & $b_{i}$ & $u_{1}$ & $u_{2}$ \\
\hline Nominal values & 85 & 300 & 80 & 52 & 42 & 432 & 221 & $9^{\circ}$ & 415 & 150 & - & $84^{\circ}$ & 400 \\
Tolerances $( \pm)$ & 0.1 & 0.3 & 0.3 & 0.35 & 0.35 & 0.3 & 0.3 & $1^{\circ}$ & 0.3 & 0.2 & 0.1 & $1^{\circ}$ & 0.25 \\
\hline
\end{tabular}

Step 1: Equivalent deviations of circularities, joint clearance and assembly deformation.

Circularities $b_{i}$ in Figure 10 are sources of deviation for this kinematic assembly as they are in the direction of deviation accumulation. These deviations are equivalent to dimensional deviations of 0 $\pm t / 2$, in which directions were along with its tolerance zone $t$. Because the areas of both surfaces in the joint are approximately equivalent, $b_{i}$ is equivalent to $0 \pm 0.05 \mathrm{~mm}$.

Virtual link models of joints are shown in Figure 11. 


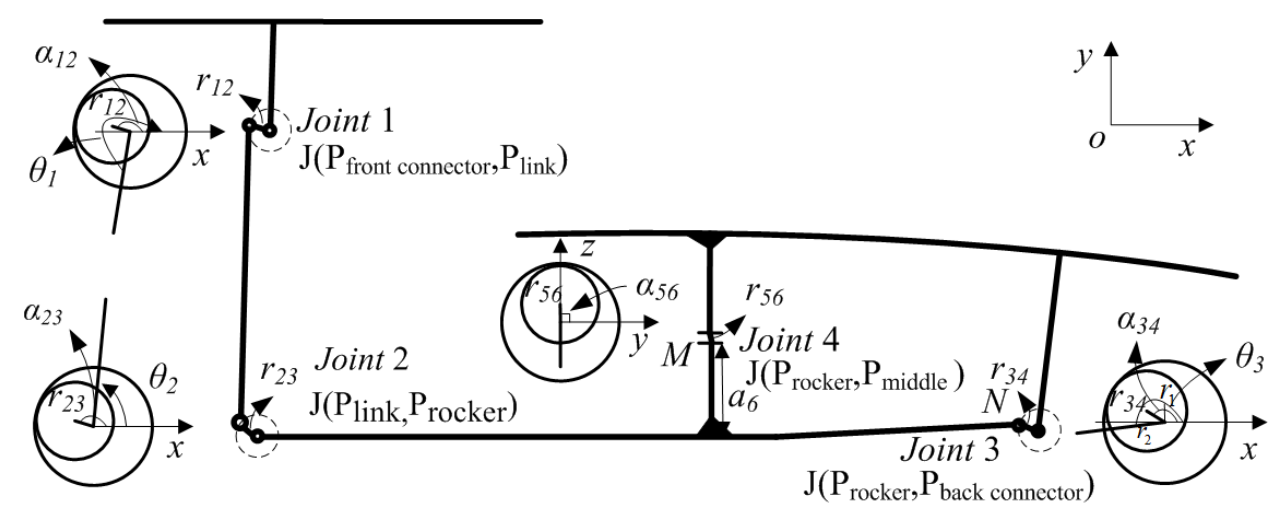

Figure 11 Virtual link model of joints in wing flap.

Joints ( $\mathrm{J}\left(\mathrm{P}_{\text {front connector, }} \mathrm{P}_{\text {link }}\right), \mathrm{J}\left(\mathrm{P}_{\text {link }}, \mathrm{P}_{\text {rocker }}\right), \mathrm{J}\left(\mathrm{P}_{\text {rocker }}, \mathrm{P}_{\text {back connector }}\right)$ and $\left.\mathrm{J}\left(\mathrm{P}_{\text {rocker }}, \mathrm{P}_{\text {middle connector }}\right)\right)$ in the kinematic assembly are denoted as Joint $i$. Dimension $r_{i j}$ and deviation $\Delta r_{i j}$ of the virtual link for Joint $i$ were determined by Eq. (1). Taking Joint 1 as an example, the initial angle $\theta_{0}$ is $240^{\circ}$ when the wing flap is folded. The orientation angle $\alpha$ is solved by Eq. (3). $\alpha_{i j}$ is solved similarly in Table 2.

Table 2 Parameters of virtual links.

\begin{tabular}{lllll}
\hline Joint & Joint 1 & Joint 2 & Joint 3 & Joint 4 \\
\hline Dimension parameters & $r_{12} / \mathrm{mm}$ & $r_{23} / \mathrm{mm}$ & $r_{34} / \mathrm{mm}$ & $r_{56} / \mathrm{mm}$ \\
Nominal values & 0.5 & 0.5 & 1.5 & 1.5 \\
Tolerances $( \pm)$ & 0.01 & 0.01 & 0.02 & 0.02 \\
Initial angle $\theta_{0} / \mathrm{deg}$ & 240 & 60 & 189 & 0 \\
Orientation angle $\alpha / \mathrm{deg}$ & 150 & 150 & 144 & 90 \\
\hline
\end{tabular}

Due to the $\mathrm{P}_{\text {rocker }}$ being a large and slender part, deformation is introduced by gravity during assembly. Therefore, assembly deformation is one of deviations in this example. The vertical deformation of point $M$ in Figure 11 is $0.52 \mathrm{~mm}$ using FEA. It has an impact on dimension $a_{6}$. Similarly, the vertical deformation of point $N$ is $0.52 \mathrm{~mm}$. This has an impact on the positional deviation of $\mathrm{P}_{\text {rocker. }}$ Then, the new dimension and tolerance of $a_{6}$ become $41 \pm 0.48 \mathrm{~mm}$ when assembly deformation is coupled, and angular deviation of $\mathrm{P}_{\text {rocker }}$ is $-0.25^{\circ}$.

Step 2: Deviation representation with vector. Deviations in kinematic assembly are unified and represented by a vector after equivalent analysis. Representation results are listed in Table 3.

Table 3 Vector representation of deviation sources.

\begin{tabular}{ccccccccc}
\hline Vector & Value $( \pm)$ & Direction $\left({ }^{\circ}\right)$ & Vector & Value $( \pm)$ & Direction $\left({ }^{\circ}\right)$ & Vector & Value $( \pm)$ & Direction $\left({ }^{\circ}\right)$ \\
\hline $\boldsymbol{a}_{1}$ & 0.1 & -90 & $\boldsymbol{a}_{2}$ & 0.3 & -120 & $\boldsymbol{a}_{3^{\prime}}$ & 0.3 & -0.25 \\
$\boldsymbol{a}_{4}$ & 0.3 & 84 & $\boldsymbol{a}_{5}$ & 0.35 & -90 & $\boldsymbol{a}_{6^{\prime}}$ & 0.48 & 90 \\
$\boldsymbol{a}_{7}$ & 0.3 & 9 & $\boldsymbol{a}_{8^{\prime}}$ & 0.3 & -0.25 & $\boldsymbol{a}_{9}$ & 0.25 & -6 \\
$\boldsymbol{a}_{10}$ & 0.2 & 174 & $\boldsymbol{b}_{1}$ & 0.05 & 150 & $\boldsymbol{b}_{2}$ & 0.05 & 150 \\
$\boldsymbol{b}_{3}$ & 0.05 & 144 & $\boldsymbol{b}_{4}$ & 0.05 & 90 & $\boldsymbol{r}_{12}$ & 0.01 & 150 \\
$\boldsymbol{r}_{23}$ & 0.01 & 150 & $\boldsymbol{r}_{34}$ & 0.02 & 144 & $\boldsymbol{r}_{56}$ & 0.02 & 90 \\
\hline
\end{tabular}


As shown in Table $3, \boldsymbol{a}_{\boldsymbol{i}}$ is the dimensional deviation vector. $\boldsymbol{b}_{\boldsymbol{i}}$ is the equivalent vector of geometric deviation $i . \boldsymbol{r}_{i j}$ is the equivalent vector of joint clearance $i . \boldsymbol{a}{ }^{\prime}$ is the new dimensional deviation vector whose deformation is considered.

Step 3: Deviation accumulation paths, vector loops and vector equation modeling. DRFs $\left(\mathrm{DRF}_{\text {rocker }}, \mathrm{DRF}_{\text {link }}, \mathrm{DRF}_{\text {wing body }}, \mathrm{DRF}_{\text {wing flap }}, \mathrm{DRF}_{\text {front connector }}, \mathrm{DRF}_{\text {middle connector }}\right.$, and $\left.\mathrm{DRF}_{\text {back connector }}\right)$ are defined according to design references and assembly locating datum. DPs from joints to DRFs are created along with the direction of dimension vector. Deviation accumulation path from $\mathrm{P}_{\text {rocker }}$ to $\mathrm{P}_{\text {back connector }}$ is shown in Figure 12. The enlarged view shows a deviation accumulation path in joint 3 which can be simplified as a dimension vector $\boldsymbol{r}_{34}$ of virtual link according to rule 3 of DPs.

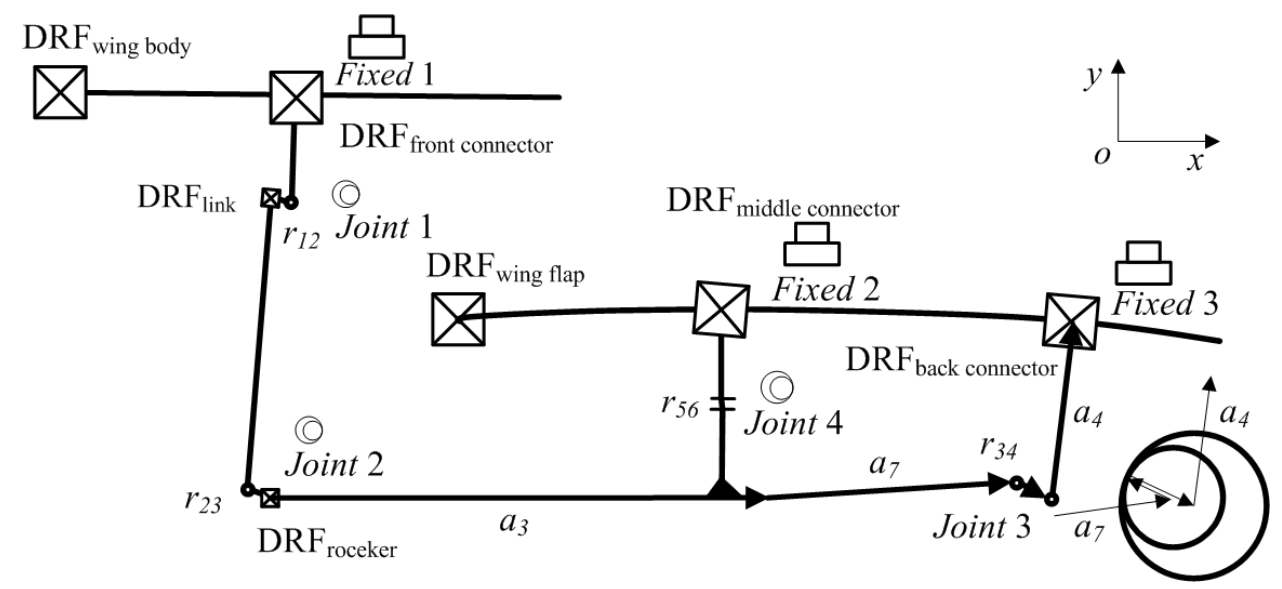

Figure 12 DRFs and deviation accumulation paths.

The closed and opened vector loops are created in Figure 13 based on deviation accumulation paths when initial angle of $\mathrm{P}_{\text {rocker }}$ is $240^{\circ}$.

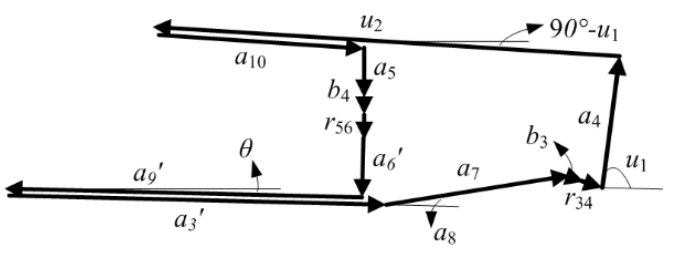

(a) The closed vector loop

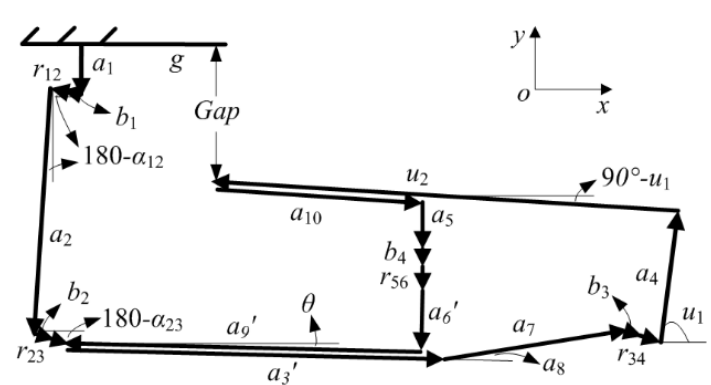

(b)The opened vector loop

Figure 13 The closed and opened vector loops.

Vector equations are generated in $S=(x, y, \theta)$, as follows: 


$$
\begin{aligned}
h_{x}= & a_{3}^{\prime} \cos \theta+a_{7} \cos \left(a_{8}+\theta\right)+r_{34} \cos \left(45-a_{8}+\theta\right)+a_{4} \cos u_{1}+u_{2} \cos \left(90-u_{1}\right)+a_{10} \cos \left(90-u_{1}\right)+a_{5} \cos (90)+ \\
& r_{56} \cos (90)+a_{6}^{\prime} \cos 90+a_{9}^{\prime} \cos 0=0 ; \\
h_{y}= & a_{3}^{\prime} \sin \theta+a_{7} \sin \left(a_{8}+\theta\right)+r_{34} \sin \left(45-a_{8}+\theta\right)+a_{4} \sin u_{1}+u_{2} \sin \left(90-u_{1}\right)+a_{10} \sin \left(90-u_{1}\right)+a_{5} \sin (90)+ \\
& r_{56} \sin (90)+a_{6}^{\prime} \sin 90+a_{9}^{\prime} \sin 0=0 ; \\
h_{\theta}= & \theta+a_{8}+\theta+45-\left(a_{8}+\theta\right)+45-\left(a_{8}+\theta\right)+u_{1}+90+180-90-0-0-0-0-\theta-180=0 \\
\text { Gap }= & g \sin (0)+a_{1} \sin (90)+r_{12} \sin \left(180-\alpha_{12}\right)+a_{2} \sin \left(\theta_{0}\right)+r_{23} \sin \left(180-\alpha_{23}\right)+a_{3}^{\prime} \sin \theta+a_{7} \sin \left(a_{8}+\theta\right)+ \\
& r_{34} \sin \left(45-a_{8}+\theta\right)+a_{4} \sin u_{1}+u_{2} \sin \left(90-u_{1}\right)
\end{aligned}
$$

The sensitivity matrix of deviation source is calculated by Eq. (18), as follow:

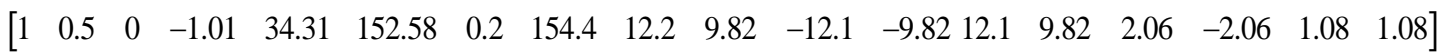

Step 4: Deviation accumulation calculation and result analysis. The functional requirement of this kinematic assembly is Gap between $\mathrm{P}_{\text {wing body }}$ to $\mathrm{P}_{\text {wing flap. The precision analysis model is }}$ established using multi-deviation sources, as shown in Table 3. Monte-Carlo simulation is completed and the result is shown in Figure $14(a)$. The probability of simulated value that falls into the requirement scope is $86.98 \%$. For comparison with other methods, DLM is also applied to deal with this problem. The simulation result is shown in Figure $14(b)$ and the accepted probability is $100 \%$.

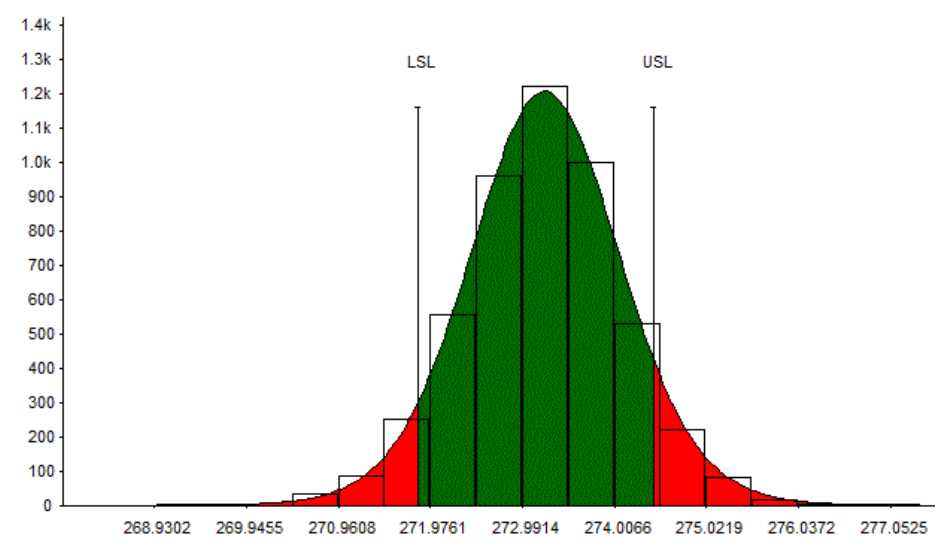

(a)

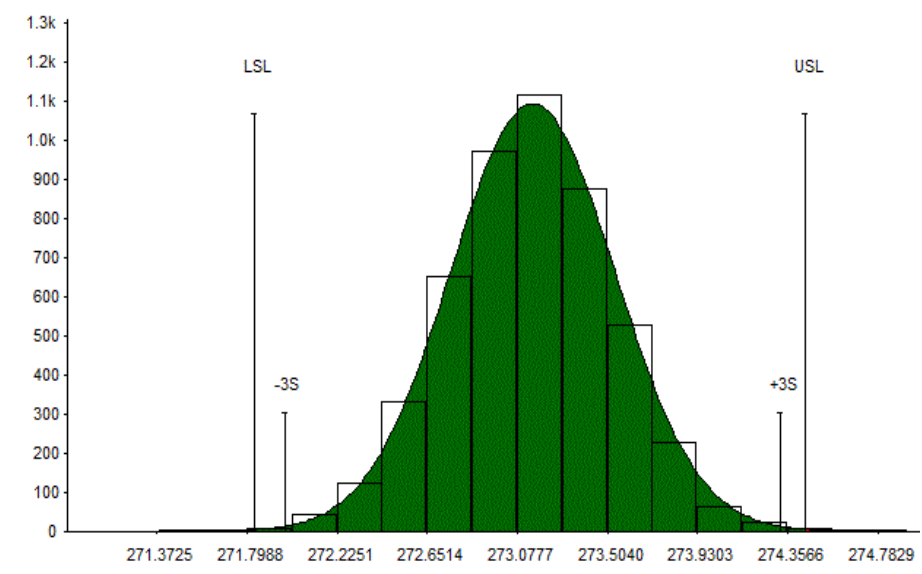

(b)

Figure 14 Simulation results of Gap between $\mathrm{P}_{\text {wing body }}$ and $\mathrm{P}_{\text {wing flap. }}$ 
The comparison of simulated results with DLM, measured value and requirement, is listed in Table 4.

Table 4 Comparison of simulation results.

\begin{tabular}{llll}
\hline simulated values & & Average value of measurement & Requirement \\
\hline The proposed method & DLM & $291.10 \mathrm{~mm}$ & $285 \pm 5 \mathrm{~mm}$ \\
\cline { 1 - 3 } $86.98 \%$ & $100 \%$ & & \\
\hline
\end{tabular}

The result of kinematic APA, considering the equivalence of multi-deviation source, shows a good conformity with the measured value. However, it does not reach the design requirement, according to 3 sigma concepts and a tolerance optimization is needed. On the other hand, the probability of simulation that falls into the requirement scope is $100 \%$ when DLM is applied. This result meets the design requirement, but the actual assembly is not qualified. This is due to DLM (or Worst-Case method) not considering joint clearance deviation and assembly deformation in deviation accumulation modeling. Hence, the proposed method provides a more accurate result for APA of kinematic mechanisms.

We have proposed a kinematic APA method, based on equivalence of multi-deviation source, and an example has been used to demonstrate the application process of this method. Compared with the existing Worst-Case method and DLM, the presented method has several advantages. Not only are part manufacturing deviations (such as dimensional and geometric form deviation) considered, but also assembly deviations (such as joint clearance, assembly deformation) are taken into account during kinematic APA. As numerous kinds of deviation sources are considered in assembly deviation modeling, this method has an accurate analysis result and is deemed suitable for the kinematic APA of complex production, such as aircrafts and motion mechanisms. It can also be used in conjunction with the DLM and can be extended to other products. If it is used in the simple rigid assembly of products, the accuracy is similar to existing methods. However, this method also has its limitations. The deviation accumulation model constructed using this method includes dimension deviation, geometry deviation, joint clearance and assembly deformation. However, it cannot express runout deviation. Moreover, this method cannot analyze assembly precision based on measured error data, as the measured error data are non-normal distribution.

When the proposed method is applied during the APA of kinematic mechanisms, there are two main difficulties. First, assembly joints usually include fixed and movable joints. The type of joint should be determined with equivalent joint clearance deviation. However, how to define joint types is dependent on user choice as joint types need to be determined according to the actual working conditions of the assembly. This may cause the accuracy of APA to be reduced. Therefore, determining the joint type requires users to be familiar with the motion of the assembly object, so as to select the correct type. Second, when the kinematic assembly is more complex and contains a greater number of joints, the parameters in the deviation accumulation equation also increase, with the equation becoming more complex, difficult to model and easier to make mistakes. In addition, the efficiency of solving the deviation accumulation equation is reduced. In 
this study, there are 4 parts, 4 joints and 18 parameters. Taylor expansion method is used to linearize the deviation accumulation equation and solve it. In future, other advanced methods can be studied to help solve this problem.

\section{Conclusion and future work}

We have proposed and validated a precision analysis method for kinematic assembly based on equivalence of deviation source. The main contributions of this research are as follows.

(1) An equivalent method of multi-deviation source is proposed based on the effect analysis of deviation on assembly position. When the influence mechanism of deviation variation on the matting position of parts is studied, the equivalent models of geometric deviation, joint clearance, assembly deformation are established. The uniform expression of multi-deviation sources information in the assembly model is achieved by using deviation vector. This equivalent method of multi-deviation source makes it possible to predict assembly precision accurately.

(2) A multi-dimensional vector loop modeling method for deviation propagation and accumulation is proposed based on assembly constraints. The multi-dimensional vector loop and vector equation of assembly deviation accumulation are constructed. Additionally, the propagation path and accumulation mechanism of APA are expressed mathematically based on the above model. Sensitivity calculation of assembly deviation sources are also formalized expression. This provides an accurate and effective means for APA and optimization.

The following presents a perspective overview of future work relating to the kinematic APA method which is considered to be challenging, but promising.

(1) The mathematic models of precision analysis, especially the deviation representation and deviation accumulation for runout, still need to be studied in more depth. In fact, a runout deviation can be seen as the combination of form deviation and positional deviation, which is more complex than dimensional and form deviation.

(2) The existing precision analysis models mainly focus on deviation propagation with series connections. However, solutions for deviation representation and accumulation with parallel connections will greatly reduce the gap between the constructed model and the actual situation. This is beyond the scope of this paper and may be considered as a suggestion for future work.

\section{Acknowledgements}

This work was partially supported by the Natural Science Basic Research Project of Shaanxi Province, China (Grant Nos. 2019JM-435 and 2019JM-073), the China Postdoctoral Science Foundation (Grant No. 2018M633439) and the Key Laboratory of Road Construction Technology and Equipment (Chang'an University), MOE (Grant No. 300102258506). 


\section{References}

Polini, W. and Corrado, A. (2015). "Geometric tolerance analysis through jacobian model for rigid assemblies with translational deviations". Assembly Automation, Vol. 36, No. 1, pp. 72-79.

Geetha, K., Ravindran, D. and Kumar, M.S. (2015). "Concurrent tolerance allocation and scheduling for complex assemblies". Robotics and Computer-Integrated Manufacturing, Vol. 35, pp. 84-95.

Sahani, A.K., Sharma, A.K. and Bajpai, J.K. (2017). "Tolerance stack up analysis of a mechanical assembly". Materials Today Proceedings, Vol. 4, No. 2, pp. 1459-1468.

Dantan, J. Y. and Qureshi, A. J. (2009). "Worst-case and statistical tolerance analysis based on quantified constraint satisfaction problems and monte carlo simulation". Computer Aided Design, Vol.41, No.1, pp. 1-12.

Zhang, G. N., Yang, Z. H., Li Q. S., et al. (2017). "A new method of tolerance analysis model based on convex hull for 3D assembly". Modular Machine Tool \& Automatic Manufacturing Technique, Vol. 2017.

Cao, Y., Liu, T., and Yang, J. (2018). "A comprehensive review of tolerance analysis models". International Journal of Advanced Manufacturing Technology, Vol. 97, No. 5-8, pp. 1-31.

Light, R., Gossard, D. (1982). "Modification of geometric models through variational geometry". Computer Aided Design, Vol. 14, No. 4, pp. 201-214.

Srinivasan, V. and Jayaraman, R. (1989). "Geometric tolerancing: II. Conditional tolerance". IBM Journal of Research \& Development, Vol. 33, No. 2, pp. 105-124.

Geis, A., Husung, S., Oberänder, A., Weber, C., and Adam, J. (2015). "Use of vectorial tolerances for direct representation and analysis in cad-systems". Procedia CIRP, Vol. 27, No. 9, pp. 230-240.

Kramer, G.A. (1993). "Solving geometric constraint systems: A case study in kinematics". Computer Aided Design, Vol. 25, No. 10, pp. 678-679.

Chase, K. W., Gao, J., and Magleby, S. P. (1995). "General 2-D tolerance analysis of mechanical assemblies with small kinematic adjustments". Journal of Design and Manufacturing, Vol. 5, pp. 263-274.

Mujezinovic, A., Davidson, J.K. and Shah J. J. (2004). "A new mathematical model for geometric tolerances as applied to polygonal faces". Journal of Mechanical Design, Vol. 126. No. 3, pp. 504-518.

Davidson, J.K., Mujezinovic, A. and Shah, J. J. (2012). "A new mathematical model for geometric tolerances as applied to round faces". Journal of Mechanical Design, Vol. 124, No. 4, pp. 609-622.

Desrochers, A. and Clémentt, A. (1994). "A dimensioning and tolerancing assistance model for CAD/CAM systems". International Journal of Advanced Manufacturing Technology, Vol. 9, No. 9, pp. 352-361.

Whitney, D.E., Gilbert, O.L. and Jastrzebski, M. (1994). "Representation of geometric variations using matrix transforms for statistical tolerance analysis in assemblies". Research in Engineering Design, Vol. 6, No. 4 pp. 191-210.

Corrado, A. and Polini, W. (2017). "Manufacturing signature in jacobian and torsor models for tolerance analysis of rigid parts". Robotics and Computer-Integrated Manufacturing, Vol. 46, No. 15-24.

Teissandier, D., Couétard, Y. and Gérard, A. (1999). "A computer aided tolerancing model: proportioned assembly clearance volume". Computer Aided Design, Vol.31, No. 13, pp. 805-817.

Peng, H. and Wang. B. (2017). "3D statistical tolerance analysis technique and the application in piston aeroengine assembly". International Conference on Mechanical and Aerospace Engineering, Vol. 2017, pp. 400-404.

D'Errico, J. R. and Zaino, N. A. J. (1998). "Statistical tolerancing using a modification of taguchi's method". Technometrics, Vol. 30, No. 4, pp. 397-405. 
Qureshi, A. J., Dantan, J. Y., Sabri, V., et al. (2012). "A statistical tolerance analysis approach for over-constrained mechanism based on optimization and monte carlo simulation". Computer Aided Design, Vol. 44, No. 2, pp. $132-142$.

Rivest, L., Fortin, C. and Morel, C. (1994). "Tolerancing a solid model with a kinematic formulation". Computer Aided Design, Vol. 26, No. 6, pp. 465-476.

Chen, H., Jin, S., Li, Z., and Lai, X. (2015). "A modified method of the unified jacobian-torsor model for tolerance analysis and allocation". International Journal of Precision Engineering and Manufacturing, Vol. 16, No. 8, pp 1789-1800.

Chase, K.W. and Parkinson, A. R. (1991). "A survey of research in the application of tolerance analysis to the design of mechanical assemblies". Research in Engineering Design, Vol. 3, No. 1, pp. 23-37.

Wittwer, J. W., Chase, K. W. and Howell, L. L. (2004). "The direct linearization method applied to position error in kinematic linkages". Mechanism and Machine Theory, Vol. 39, No. 7, pp. 681-693.

Sacks, E. and Joskowicz, L. (1998). "Parametric kinematic tolerance analysis of general planar systems". Computer Aided Design, Vol. 30, No. 9, pp. 707-714.

Muvengei, O., Kihiu, J. and Ikua, B. (2011). "Dynamic analysis of multi-body mechanical systems with imperfect kinematic joints: A literature survey and review". Sustainable Research \& Innovation Proceedings, 2011. Vol. 3.

Bruyère, J., Dantan, J.Y., Bigot, R., et al. (2007). "Statistical tolerance analysis of bevel gear by tooth contact analysis and monte carlo simulation". Mechanism and Machine Theory, Vol. 42, No. 10, pp. 1326-1351.

Parenticastelli, V. and Venanzi, S. (2005). "Clearance influence analysis on mechanisms". Mechanism \& Machine Theory, Vol. 40, No. 12, pp. 1316-1329.

Parenticastelli, V., Venanzi, S. (2005). "A new technique for clearance influence analysis in spatial mechanisms". Journal of Mechanical Design, Vol. 127, No. 3, pp. 446-455.

Schwab, A. L., Meijaard, J.P. and Meijers, P. (2002). "A comparison of revolute joint clearance models in the dynamic analysis of rigid and elastic mechanical systems". Mechanism and Machine Theory, Vol. 37, No. 9, pp. 895-913.

Beaucaire, P., Gayton, N., Duc, E. and Dantan, J. Y. (2013). "Statistical tolerance analysis of a mechanism with gaps based on system reliability methods". Procedia CIRP, Vol. 10, pp. 2-8.

Corrado, A., Polini, W., Moroni, G. and Petròet S. (2018). "A variational model for 3D tolerance analysis with manufacturing signature and operating conditions". Assembly Automation, Vol. 38, pp.10-19.

Tsai, M. J., Lai, T. H. (2008). "Accuracy analysis of a multi-loop linkage with joint clearances". Mechanism and Machine Theory, Vol. 43, No. 9, pp. 1141-1157.

Polini, W. (2014). "To model joints with clearance for tolerance analysis". Proceedings of the Institution of Mechanical Engineers, Part B: Journal of Engineering Manufacture, Vol. 228, No. 12, pp. 1689-1700.

Ting, K. L., Zhu, J. and Watking, D. (2000). "The efects of joint clearance on position and orientation deviation of linkages and manipulators". Mechanism and Machine Theory, Vol. 35, No. 3, pp. 391-401.

Tsai, M. J., Lai, T. H. (2004). "Kinematic sensitivity analysis of linkage with joint clearance based on transmission quality". Mechanism and Machine Theory, Vol. 39, No. 11, pp. 1189-1206.

Rhyu, J. H. and Kwak, B. M. (1998). "Optimal stochastic design of four-bar mechanisms for tolerance and clearance". Journal of Mechanical Design, Vol. 110, No. 3, pp. 255-262.

Erkaya, S. and İbrahim, U. (2009). "Determining link parameters using genetic algorithm in mechanisms with joint clearance". Mechanism and Machine Theory, Vol. 44, No. 44, pp. 222-234. 
Wu, W., Rao, S. S. (2004). "Interval approach for the modeling of tolerances and clearances in mechanism analysis". Journal of Mechanical Design, Vol. 126, No. 4, pp. 581-592.

Mallik, A. K., Dhande, S. G. (1987). "Analysis and synthesis of mechanical error in path-generating linkages using a stochastic approach". Mechanism and Machine Theory, Vol. 22, No. 2, pp. 115-123.

Lee, S. J., Gilmore, B. J. (1991). "The determination of the probabilistic properties of velocities and accelerations in kinematic chains with uncertainty". Journal of Mechanical Design, Vol. 113, No. 1, pp. 84-90.

Zhao, Q. Q., Guo, J. K., Hong, J. (2018)."Uncertainty analysis of assembly error of planar single-loop mechanisms based on the rotatability laws of linkages". Journal of Mechanical Engineering, Vol. 54, No. 11, pp. 29-38.

Walter, M., Sprügel, T., and Wartzack, S. (2013). "Tolerance analysis of systems in motion taking into account interactions between deviations". Proceedings of the Institution of Mechanical Engineers, Part B: Journal of Engineering Manufacture, Vol. 227, No. 5 pp. 709-719.

Lee, C.L., Tang. G. R. (2000). "Tolerance design for products with correlated characteristics". Mechanism and Machine Theory, Vol. 35, No. 12, pp. 1675-1687.

Dantan, J. Y., Bruyere, J., Vincent, J. P., and Bigot, R. (2008). "Vectorial tolerance allocation of bevel gear by discrete optimization". Mechanism and Machine Theory, Vol. 43, No. 11, pp. 1478-1494.

Gerbino, S., Patalano, S. and Franciosa, P. (2008). "Statistical variation analysis of multi-station compliant assemblies based on sensitivity matrix". International Journal of Computer Applications in Technology, Vol. 33, No. 1, pp. 12-23.

Xie, K., Wells, L., Camelio, J. A., and Youn, B. D. (2007).. "Variation propagation analysis on compliant assemblies considering contact interaction". Journal of Manufacturing Science and Engineering, Vol. 129, No. 5, pp. 934-942. Dupac, M., Beale, D.G. (2010). "Dynamic analysis of a flexible linkage mechanism with cracks and clearance". Mechanism and Machine Theory, Vol. 45, No. 12, pp. 1909-1923.

Stuppy, J., Meerkamm, H. (2009). "Tolerance analysis of a crank mechanism by taking into account different kinds of deviation". In: Proceedings of the 11th CIRP international seminar on computer aided tolerancing.

Imani, B.M., Pour, M. (2009). "Tolerance analysis of flexible kinematic mechanism using DLM method". Mechanism and Machine Theory, Vol. 44, No. 2, pp. 445-456.

Wu, Y., Chen. C. (2018). "An automatic generation method of the coordinate system for automatic assembly tolerance analysis". International Journal of Advanced Manufacturing Technology, Vol. 95, No. 1-4, pp. 889-903.

Schleich, B., Anwer, N., Mathiey, L. et al. (2014). "Skin Model Shapes: A new paradigm shift for geometric variations modeling in mechanical engineering". Computer Aided Design, Vol. 50, No. 3, pp. 1-15.

Schleich, B., Wartzack, S. (2014). "A discrete geometry approach for tolerance analysis of mechanism". Mechanism and Machine Theory, Vol. 77, No. 7, pp. 148-163.

Franciosa, P., Gerbino, S. and Patalano, S. (2011). "Simulation of variational compliant assemblies with shape errors based on morphing mesh approach". International Journal of Advanced Manufacturing Technology, Vol. 53, No. $1-4$, pp. 47-61.

Goka, E., Homri, L., et al. (2019) "Statistical tolerance analysis of over-constrained mechanical assemblies with form defects considering contact types". Journal of Computing and Information Science in Engineering, Vol. 19, No.2, 021010. 